\title{
Physical activity, sedentary behaviour, and sleep: movement behaviours in early life
}

Anthony D Okely ${ }^{1}$, Mark S Tremblay ${ }^{2}$, John J Reilly ${ }^{3}$, Catherine E Draper ${ }^{4,5}$, \& Fiona Bull ${ }^{6}$

${ }^{1}$ Early Start, Faculty of Social Sciences, University of Wollongong, Australia.

${ }^{2}$ Children's Hospital of Eastern Ontario Research Institute, Ottawa, Canada.

${ }^{3}$ University of Strathclyde, Physical Activity for Health Group, School of Psychological Sciences and Health, , Glasgow, Scotland.

${ }^{4}$ MRC/Wits Developmental Pathways for Health Research Unit, University of the Witwatersrand, South Africa.

${ }^{5}$ Division of Exercise Science and Sports Medicine, University of Cape Town, South Africa.

${ }^{6}$ Surveillance \& Population Based Prevention, is Health Organization, Geneva.

By the time they reach the age of 5 years, around 41 million of the world's children will be overweight or obese with this number likely to increase to at least 50 million by $2030^{1}$. While prevalence rates among children under the age of 5 have plateaued in some developed countries albeit at a high prevalence - rates have increased in recent years in low- and middle-income countries ${ }^{2}$. Once a child is obese, even at this young age, it is likely to persist resulting in a constellation of health and social consequences across the life course. Prevention of excess weight gain early in life provides the most cost-effective and ethical approach to addressing the obesity crisis and consequent high rates of non-communicable diseases (NCDs).

In response to this epidemic, the World Health Organization's Commission on Ending Childhood Obesity (WHO ECHO) ${ }^{3}$ recognised the need to focus on prevention in the early years (ages $0-5$ ) and for this focus to move beyond personal choice to parents and society creating the opportunities, values, and environments to support healthy diet and movement behaviours (physical activity, sedentariness, and sleep).

The WHO ECHO Report highlighted the importance of promoting sleep and physical activity and reducing sedentary time in the fight to reduce the prevalence of obesity in early childhood, and to help tackle the NCD crisis ${ }^{3}$. This focus on movement behaviours across the 24 -hour period is a positive step, recognising that the whole day matters and individual movement behaviours such as physical activity, sedentary behaviour, and sleep need to be considered in relation to each other when examining their associations with obesity and other indicators of health in children. Canada and Australia have developed 24-Hour Movement Guidelines for the Early Years that were coreleased in November $2017^{4,5}$. The WHO is also developing similar movement guidelines for the early years to be released in 2018. The new guidelines provide the global community with evidencebased guidance on recommended levels of physical activity, sedentary time and sleep. The United Kingdom and South Africa are also developing similar guidelines. This is especially significant in South Africa, which to our knowledge is the first low- or middle-income country to adopt this 
preventive, integrated approach. Having guidelines more widely available internationally will increase momentum for promotion of healthier movement behaviour patterns in young children, facilitate better monitoring and surveillance of these behaviours, and further our understanding of trends, inequalities and determinants, which will better inform subsequent efforts for improvement. Regular monitoring and surveillance has been occurring for some time in the nutrition area through the annual Global Nutrition Report which is facilitated through the collection of nutrition data on young children in a large number of countries (140 in the 2017 report) ${ }^{6}$. We call on such countries to do the same for movement behaviours.

Work has also focused on linking physical activity to the achievement of specific Sustainable Development Goals (SDG) through the Bangkok Declaration on Physical Activity for Global Health and Sustainable Development ${ }^{7}$. This Declaration identified opportunities for promoting physical activity in the early years, primarily through SDG 4 (quality education) and SDG 11 (inclusive, safe, resilient and sustainable cities and communities) and motivated the development of the WHO Global Action Plan on Physical Activity (GAPPA) ${ }^{8}$. Recommended for endorsement at the $142^{\text {nd }}$ session of the WHO Executive Board in January 2018, the GAPPA provides a framework for 20 multidimensional policy actions, each of which will play an important role in promoting healthy movement behaviours, and result in the achievement of four strategic objectives to create active: 1) societies; 2) environments; 3 ) people; and 4) systems. While the objectives are designed to not be age-specific, at least 11 of the proposed actions for member states, the WHO Secretariat, and other stakeholders do specifically target the early years.

What else needs to be done over the next 10 years to promote healthy movement behaviours to prevent obesity and promote healthy development in young children? First, as a foundation, is the collection of international surveillance data on movement behaviours. Currently, the youngest age for such global data is 13 years as part of the Global School-based Student Health Survey (GSHS). These data should be routinely collected, mapping changes against changes in child obesity and other health and developmental outcomes, helping to identify key catalysts of change and where to start intervening. Second, the two Lancet series on Physical Activity in 2012 and 2016 provided much needed epidemiological evidence on the importance of physical activity and how to promote it, including a call for global action on physical activity policy. However, neither of these series had a strong focus on children, nor addressed the full complement of movement behaviours. The third series in 2020 provides an opportunity to address these limitations and in particular to report on progress that has been made since 2016 in the area of physical activity and sedentary behaviour in young children, especially in response to the WHO ECHO Report, Bangkok Declaration, and WHO GAPPA, and especially in low- and middle-income countries. At present, very little is 
known about the levels of inequalities in, and determinants of, movement behaviours in these countries. This is concerning, recognizing changes occurring in many of these countries that are anticipated to negatively impact on movement behaviours. For example, rapid urbanisation resulting in high levels of air pollution, increased motorised transportation, and poorer walkability in high population dense cities make outdoor physical activity less safe for children. Sleep duration and quality may also be compromised in settings where population density is high as a result of sharing of rooms and beds or digital screens in bedrooms. Many families transitioning to cities also desire for their children technological lifestyles seen in developed countries, resulting in children accumulating large amounts of sedentary screen time, possibly displacing active play while increasing exposure to marketing of unhealthy foods. Third, similar to INFORMAS for nutrition (International Network for Food and Obesity/NCD Research Monitoring and Action Support) ${ }^{9}$, there is a need for a global physical activity network to monitor physical activity environments, policies and systems to describe societal and commercial determinants, inform policy specifications, and hold societies accountable.

Much progress has been made recently to highlight the importance of 24-hour movement behaviours in young children. Investment needs to be continued if efforts to prevent obesity, promote healthy active living, and respect the child's right to active play are to be realised.

\section{Acknowledgements}

We thank Bridget Kelly for her critical feedback on this commentary. 


\section{References}

1. UNICEF, WHO, Bank W. Levels and trends in child malnutrition: UNICEF/WHO/World Bank joint child malnutrition estimates. Key findings of the 2017 edition, UNICEF, New York; WHO, Geneva; World Bank, Washington DC; 2017.

2. Abarca-Gómez L, Abdeen ZA, Hamid ZA, et al. Worldwide trends in body-mass index, underweight, overweight, and obesity from 1975 to 2016: a pooled analysis of 2416 population-based measurement studies in 128.9 million children, adolescents, and adults. The Lancet. 2017; 390(10113):2627-2642.

3. World Health Organization. Report of the commission on ending childhood obesity, Geneva, Switzerland, WHO; 2016.

4. Okely AD, Ghersi D, Hesketh K, et al. Using the GRADE-ADOLOPMENT approach to produce the Australian 24-hour movement guidelines for the early years: An Integration of Physical Activity, Sedentary Behaviour, and Sleep. BMC Public Health. 2017; in press.

5. Tremblay MS, Carson V, Chaput JP, et al. Canadian 24-Hour Movement Guidelines for the Early Years (0-4 years): An Integration of Physical Activity, Sedentary Behaviour, and Sleep. BMC Public Health. 2017; this issue.

6. Development Initiatives. Global Nutrition Report 2017: Nourishing the SDGs. Bristol, UK2017.

7. The Bangkok Declaration on Physical Activity for Global Health and Sustainable Development. The 6th ISPAH International Congree on Physical Activity and Public Health. Bangkok, Thailand: 16-19 November 2016; 2016.

8. World Health Organisation. Physical activity for health. More active people for a healthier world: draft global action plan on physical activity 2018-2030. WHO Discussion Paper. 2017, December.

9. Swinburn B, Sacks G, Vandevijvere S, et al. INFORMAS (International Network for Food and Obesity/non - communicable diseases Research, Monitoring and Action Support): overview and key principles. Obesity reviews. 2013; 14(S1):1-12.

Introduction to the Global Matrix 2.0 Report Card on the physical activity of children and youth comparing 38 countries. MS Tremblay, JD Barnes, SA Gonzalez, PT Katzmarzyk, V Onywera, JJ Reilly, GR Tomkinson. J Phys Act \& Health 2016; 13 suppl 2 s85-86. 